\title{
CheckMate—has nivolumab beaten melanoma?
}

The use of anti-PD-1 antibodies to break immunological self-tolerance has shown impressive and broad-ranging antitumour responses in patients with melanoma, kidney cancer, and non-small-cell lung cancer. The results of a recent phase I/II study demonstrated that patients with disease progression after previous ipilimumab therapy had high rates of long-term responses following nivolumab treatment. Moreover, nivolumab was

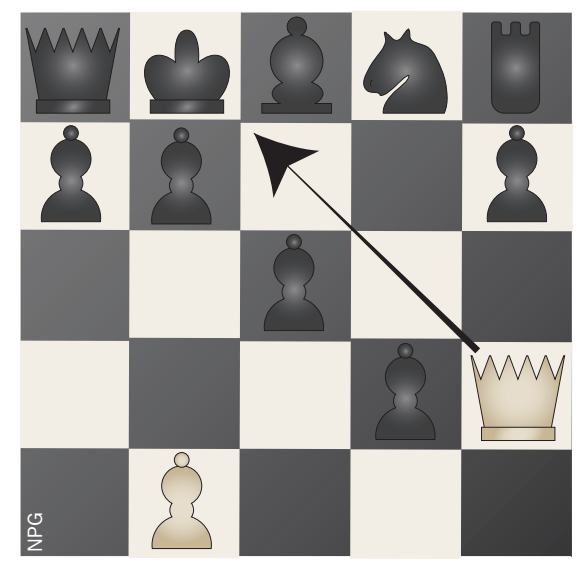

well tolerated, even after patients had experienced considerable adverse events from previous ipilimumab therapy. This prompted Jeffrey Weber and colleagues to conduct a randomized, controlled, open-label, phase III trial in patients with metastatic melanoma. This registration trial (CheckMate 037) assessed nivolumab as a single agent in an ipilimumabrefractory population, which included a BRAF-inhibitor-refractory group with $B R A F$-mutant tumours. Hence, the trial required a control arm that consisted of either dacarbazine only or paclitaxel plus carboplatin-based chemotherapy.

Confirmed objective responses at 24 weeks follow up were reported in 38 of 120 patients in the nivolumab arm $(31.7 \%)$ versus five of 47 patients (10.6\%) in the chemotherapy arm. No differences in adverse events were noted in patients receiving either dacarbazine or paclitaxel plus carboplatin. Grade 3-4 serious adverse effects were observed in 5\% of patients in the nivolumab arm and $9 \%$ of patients treated with chemotherapy.
As Weber highlights: "the most important findings were that the overall response rate of the anti-PD-1 antibody nivolumab was superior to that of the chemotherapy group, with an excellent duration of response. Furthermore, the toxicity in patients receiving nivolumab was less than for those receiving chemotherapy." The results of this study provided clear evidence of the clinical benefit of nivolumab, which led to its recent approval; nivolumab represents a new treatment option in a population with a high unmet need. Weber comments on future steps with testing immunotherapies: "the next step is to test the safety and efficacy of combination regimens with other biological compounds and sequential treatment with ipilimumab."

Lisa Hutchinson

Original article Weber, J. S. et al. Nivolumab versus chemotherapy in patients with advanced melanoma who progressed after anti-CTLA-4 treatment (CheckMate 037): a randomised, controlled, open-label, phase 3 trial. Lancet Oncol. doi:10.1016/S1470-2045(15)70076-8 